2002 99: 4357-4363

doi:10.1182/blood.V99.12.4357

\title{
High incidence of cytomegalovirus infection after nonmyeloablative stem cell transplantation: potential role of Campath-1H in delaying immune reconstitution
}

Suparno Chakrabarti, Stephen Mackinnon, Raj Chopra, Panagiotis D. Kottaridis, Karl Peggs, Peter O'Gorman, Ronjon Chakraverty, Timothy Marshall, Husam Osman, Premini Mahendra, Charles Craddock, Herman Waldmann, Geoff Hale, Christopher D. Fegan, Kwee Yong, Anthony H. Goldstone, David C. Linch and Donald W. Milligan

Updated information and services can be found at:

http://bloodjournal.hematologylibrary.org/cgi/content/full/99/12/4357

Articles on similar topics may be found in the following Blood collections:

Transplantation (1286 articles)

Clinical Trials and Observations (2398 articles)

Neoplasia (4075 articles)

Information about reproducing this article in parts or in its entirety may be found online at:

http://bloodjournal.hematologylibrary.org/misc/rights.dtl\#repub_requests

Information about ordering reprints may be found online at:

http://bloodjournal.hematologylibrary.org/misc/rights.dtl\#reprints

Information about subscriptions and ASH membership may be found online at:

http://bloodjournal.hematologylibrary.org/subscriptions/index.dtl

Blood (print ISSN 0006-4971, online ISSN 1528-0020), is published semimonthly by the American Society of Hematology, 1900 M St, NW, Suite 200, Washington DC 20036.

Copyright 2007 by The American Society of Hematology; all rights reserved.

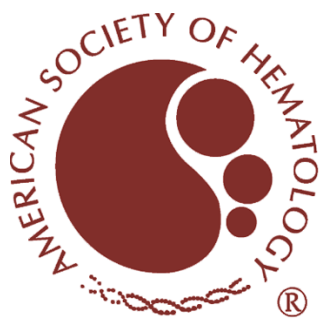




\title{
High incidence of cytomegalovirus infection after nonmyeloablative stem cell transplantation: potential role of Campath-1H in delaying immune reconstitution
}

\author{
Suparno Chakrabarti, Stephen Mackinnon, Raj Chopra, Panagiotis D. Kottaridis, Karl Peggs, Peter O'Gorman, Ronjon Chakraverty, \\ Timothy Marshall, Husam Osman, Premini Mahendra, Charles Craddock, Herman Waldmann, Geoff Hale, Christopher D. Fegan, \\ Kwee Yong, Anthony H. Goldstone, David C. Linch, and Donald W. Milligan
}

\begin{abstract}
Nonmyeloablative conditioning is increasingly used for transplantation in a wide range of diseases, but little is known about its impact on the incidence of infections and immune reconstitution. We examined the pattern and outcome of cytomegalovirus (CMV) infections monitored by polymerase chain reaction-based assays and treated preemptively in 101 patients following nonmyeloablative conditioning containing in vivo Campath-1H. Fifty-one patients (50\%) had a CMV infection at a median of 27 days after transplantation with a probability of
\end{abstract}

$84.8 \%$ in patients at risk of $\mathrm{CMV}$ infection. The probability of recurrence of CMV infection before and after 100 days was $53.6 \%$ and $46.6 \%$, respectively, and was more common in unrelated donor transplant recipients. All 3 patients who developed CMV disease died of this complication. The 2 patients with late CMV disease had grade III to IV graft-versus-host-disease (GVHD), which occurred de novo in only $4 \%$ of patients and in another $10 \%$ following donor lymphocyte infusions. The median time to $\mathrm{CD}^{+}$T-cell count more than $200 / \mu \mathrm{L}$ was 9 months in the $\mathbf{4 8}$ patients studied. The probabilities of overall survival and nonrelapse mortality at 18 months were $65 \%$ and $27.8 \%$, respectively, with no significant difference in survival between CMV-infected and -uninfected patients. The use of Campath-1H appeared to be associated with a low incidence of GVHD but a high incidence of CMV infections and prolonged immune paresis. (Blood. 2002;99:4357-4363)

() 2002 by The American Society of Hematology

\section{Introduction}

Cytomegalovirus (CMV) infection is still a major concern following allogeneic hematopoietic transplants because CMV pneumonia is fatal in $70 \%$ of patients, even when treated with a combination of antivirals and CMV hyperimmune immunoglobulin. ${ }^{1}$ While the therapeutic strategy has moved to prophylaxis with ganciclovir (GCV) or polymerase chain reaction (PCR) or antigenemia-guided preemptive therapy, ${ }^{2-4}$ the epidemiology of CMV disease has changed as well. The incidence of CMV disease beyond 100 days is increasingly observed following such preventive approaches. ${ }^{5,6}$ Chronic graft-versus-host-disease (GVHD) and prolonged use of GCV have been recognized as the 2 major risk factors for CMV disease after 100 days. ${ }^{5,6}$

The major determinants for the development of CMV disease are the type of transplant, GVHD, and its therapy. ${ }^{5-9}$ Persistent $\mathrm{CD}^{+}$lymphopenia has been identified as an important prognostic factor for development of CMV disease after antiviral therapy. ${ }^{10} \mathrm{Li}$ et al have demonstrated a strong association between the lack of $\mathrm{CMV}$-specific $\mathrm{CD}^{+}{ }^{+}$cytotoxic $\mathrm{T}$ lymphocytes and the development of CMV disease after transplantation. ${ }^{11}$

The achievement of engraftment following nonmyeloablative conditioning has widened the scope of allogeneic transplants to patients who were previously considered ineligible or at a high risk of mortality following conventional transplants. ${ }^{12-17}$ However, the use of nonmyelo- ablative conditioning in human studies has not resulted in a low incidence of GVHD as expected from preclinical studies and GVHD remains the predominant cause of death after such transplants. ${ }^{13,15-17}$

In a multicenter study from the United Kingdom, we have reported a low incidence of GVHD with the addition of Campath-1H in vivo to the conditioning regimen of fludarabine and melphalan. ${ }^{14}$ However, we also noted a high incidence of CMV reactivation in patients treated with this schedule. We report here the pattern of CMV reactivation and outcome using PCR-guided preemptive antiviral therapy and the incidence of opportunistic infections in 101 patients undergoing nonmyeloablative transplantation in this multicenter study. The T-cell subset recovery was also longitudinally monitored in 48 of these patients.

\section{Patients and methods}

\section{Patients}

Between June 1997 and February 2001, 101 patients were enrolled in this study from 4 major collaborating centers. The eligibility criteria for this study have been previously described and the study design was approved by local ethics committee of all the participating centers. ${ }^{15}$
From the Departments of Haematology and Public Health Laboratories, Birmingham Heartlands Hospital, Department of Public Health and Epidemiology, Institute of Cancer Studies, University of Birmingham, Birmingham, United Kingdom; Department of Haematology, University College Hospital, London, United Kingdom; Department of Haematology, Christie Hospital, Manchester University Hospital, Birmingham, United Kingdom; and Sir William Dunn School of Pathology, Oxford, United Kingdom.

Submitted August 23, 2001; accepted February 7, 2002.
Reprints: Donald W. Milligan, Department of Haematology, Birmingham Heartlands Hospital, Birmingham, B9 5SS, United Kingdom; e-mail: d.w.milligan@bham.ac.uk.

The publication costs of this article were defrayed in part by page charge payment. Therefore, and solely to indicate this fact, this article is hereby marked "advertisement" in accordance with 18 U.S.C. section 1734.

(C) 2002 by The American Society of Hematology 


\section{Conditioning regimen}

Conditioning treatment consisted of Campath-1H (the IgG1 humanized monoclonal antibody against CD52) $20 \mathrm{mg} / \mathrm{d}$ on days -8 to -4 , fludarabine $30 \mathrm{mg} / \mathrm{m}^{2}$ from days -7 to -3 , and melphalan $140 \mathrm{mg} / \mathrm{m}^{2}$ on day $-2 .{ }^{15}$ Cyclosporin A was started from day -1 as GVHD prophylaxis. Patients received unmanipulated peripheral blood stem cells from a matched family donor or unmanipulated bone marrow from an unrelated donor (UD).

Donor lymphocyte infusion (DLI) was not a part of the protocol and was at the discretion of the treating physician. The usual indications were persistent mixed chimerism or worsening donor chimerism and persistence, progression, or relapse of the disease that warranted the transplant.

\section{CMV surveillance}

Pretransplant serum samples from all patients and donors were tested for serologic evidence of past infection with CMV by an enzyme-linked immunosorbent assay. CMV seropositive recipients (R) or those receiving grafts from CMV seropositive donors (D) were defined as being at risk for CMV disease. Among the patients at risk for CMV disease, CMV seropositive recipients receiving graft from a $\mathrm{CMV}$-seropositive $\left(\mathrm{R}^{+} / \mathrm{D}^{+}\right)$or seonegative donor $\left(\mathrm{R}^{+} / \mathrm{D}^{-}\right)$were defined as high risk (CMV-HR) and $\mathrm{CMV}$-seronegative patients receiving transplants from seropositive donors $\left(\mathrm{R}^{-} / \mathrm{D}^{+}\right)$were termed as intermediate risk (CMV-IR). CMV seronegative patients receiving grafts from CMV-seronegative donors were considered low-risk for CMV disease (CMV-LR). CMV-LR patients received $\mathrm{CMV}^{-}$ blood products, whereas CMV-HR and CMV-IR patients were given unscreened blood products.

All the CMV-HR patients were screened for CMV reactivation by PCR-based assays on whole blood collected in EDTA. Three centers used qualitative PCR for CMV surveillance. This was done using a nested PCR in one center ${ }^{18}$ and a nonnested assay (Roche, Amplicor) in the other 2. The fourth center used the Taqman quantitative CMV PCR assay format. ${ }^{19}$ The limit of detection for this assay was defined as $500 \mathrm{CMV}$ genome copies $/ \mathrm{mL}$.

The patients were screened every week from transplantation to 100 days after the procedure. Generally those patients who had CMV infection before 100 days or had GVHD were screened for 180 days or beyond. Patients with persistent infection or disease or GVHD were screened beyond this period at the discretion of the individual center. In addition, 25 CMV-LR patients were screened for 100 days after transplantation.

\section{Preemptive antiviral therapy}

The patients received antiviral therapy if 2 consecutive qualitative PCR assays were positive or CMV genome copies exceeded $500 / \mathrm{mL}$ on 2 consecutive quantitative assays. GCV $5 \mathrm{mg} / \mathrm{kg}$ twice a day or foscarnet 120 to $180 \mathrm{mg} / \mathrm{d}$ in 2 or 3 divided doses was considered as the first-line therapy. Dose adjustments were made for impairment of renal function. Treatment was stopped if 2 consecutive nonnested or one nested PCR assay was negative or below the detection limit on 2 consecutive quantitative assays. Treatment was switched from one drug to the other if there was serious nonoverlapping toxicity. This was also instituted if there was persistent infection after 2 to 3 weeks treatment or if the infection recurred within 2 weeks after obtaining 2 negative results on PCR. Two patients from one of the centers received cidofovir as the first-line therapy as a part of a local study protocol.

\section{Definitions}

CMV infection was defined as PCR positivity on 2 consecutive assays, without clinical signs or symptoms. Recurrence of CMV infection was defined as CMV infection occurring after negative PCR assays following treatment of the initial episode of infection. CMV disease was defined as demonstration of CMV by culture or early antigen detection tests in bronchoalveolar lavage specimen in the presence of recent respiratory signs and symptoms with pulmonary infiltrates on radiology, or histologic evidence of CMV from other visceral sites. CMV infection and disease were defined as late if they occurred after 100 days. CMV-related death was defined as definite if death occurred within 6 weeks of the diagnosis of CMV disease, unrelated to the underlying disease or another etiology. Short of tissue diagnosis, death in a patient with a persistently positive PCR with no other obvious cause and presence of clinical signs or symptoms, which could be explained by CMV disease, was considered possible CMV-related death.

\section{Treatment of CMV disease}

Disease was treated with either GCV $5 \mathrm{mg} / \mathrm{kg}$ twice a day or foscarnet at a dose of up to $180 \mathrm{mg} / \mathrm{d}$ in divided doses or a combination of both. Intravenous immunoglobulin was also added to the antiviral regimen.

\section{Supportive care}

Antimicrobial prophylaxis consisted of acyclovir and fluconazole or itraconazole from the beginning of conditioning treatment. Every patient received Pneumocystis carinii prophylaxis with co-trimoxazole $480 \mathrm{mg}$ every 12 hours 3 times weekly, after the absolute neutrophil count exceeded $1.0 \times 10^{9} / \mathrm{L}$. Febrile neutropenic patients were treated with broad-spectrum antibiotics according to institutional policy of each center. Granulocyte colony-stimulating factor was administered subcutaneously at the discretion of the physician doing the transplant procedure to hasten neutrophil engraftment. Irradiated red blood cell and platelet transfusions were administered to maintain hemoglobin and platelets above $9 \mathrm{~g} / \mathrm{dL}$ and $10 \times 10^{9} / \mathrm{L}$, respectively.

\section{Estimation of T-lymphocyte subsets}

Peripheral blood was collected in EDTA for T-lymphocyte subset analysis, at least once every 3 months after the transplantation. T-lymphocyte subsets were analyzed by flow cytometry using a FACScan and SimulSET software (Becton Dickinson, Oxford, United Kingdom) as previously described. ${ }^{20}$

\section{Statistical analysis}

The primary endpoint of this study was CMV infection. Recurrence of CMV infection was evaluable only in patients followed for at least 4 weeks after remission of the first infection. Patients undergoing CMV screening beyond 100 days were evaluable for late reactivation. Data were censored at the time of death, retreatment of relapsed disease with cytotoxic drugs, death, or receipt of CMV-specific cytotoxic CD8 ${ }^{+} \mathrm{T}$ cells $(\mathrm{n}=6)$. The variables were analyzed by either $2 \times 2$ contingency tables or Student $t$ test (for continuous variables). Probabilities of CMV infection and recurrence, overall survival, and nonrelapse mortality were analyzed by the KaplanMeier method and the difference between the groups was compared using $\log$-rank $\chi^{2}$ test. The effect of the variables on time-to-CMV recurrence was tested in a Cox regression model, using the SPSS version 9 (SPSS UK, Worthing, United Kingdom). The variables examined were age, sex, disease type, donor type, CMV risk status, neutrophil engraftment, $\mathrm{CD} 4^{+} \mathrm{T}$-cell counts at 3 and 6 months, duration of previous episodes of CMV infection, GVHD, and duration of antiviral treatment. The variables were analyzed in an univariate model and the variables with $P<.10$ were entered in the multivariate model. Other outcomes (nonrelapse mortality and overall survival) were also examined in a Cox regression model, with CMV infection and recurrence as covariates.

\section{Results}

\section{Patient characteristics}

Detailed characteristics of this patient cohort are shown in Table 1. In brief, 101 patients were studied with a median follow-up of 15 months (range, 2-46 months). The median age was 44 years (range, 18-59 years). Thirteen patients received transplants for acute leukemia, 10 for chronic leukemias, and 78 for lymphoproliferative disorder or myeloma. Thirty-four patients had previous transplants (33 autografts, 1 allograft). Thirty-four patients received a bone 
Table 1. Characteristics of patients receiving nonmyeloablative transplants

\begin{tabular}{|c|c|c|c|}
\hline & $\begin{array}{l}\text { CMV-HR } \\
(n=52)\end{array}$ & $\begin{array}{l}\text { CMV-IR } \\
(\mathrm{n}=8)\end{array}$ & $\begin{array}{l}\text { CMV-LR } \\
(\mathrm{n}=41)\end{array}$ \\
\hline Age (y) median (range) & $46.5(25-59)$ & $41(21-50)$ & $42(18-59)$ \\
\hline Sex (male/female) & $33 / 19$ & $6 / 2$ & $29 / 12$ \\
\hline \multicolumn{4}{|l|}{ Disease } \\
\hline AML/ALL/MDS & $7 / 0 / 0$ & $1 / 0 / 0$ & $1 / 2 / 2$ \\
\hline CML/CLL & $4 / 3$ & $1 / 0$ & $0 / 2$ \\
\hline $\mathrm{NHL} / \mathrm{HD}$ & $19 / 5$ & $2 / 2$ & $19 / 8$ \\
\hline Myeloma & 14 & 2 & 7 \\
\hline Previous transplants & 13 & 4 & 17 \\
\hline \multicolumn{4}{|l|}{ Donor type } \\
\hline Matched family & 36 & 5 & 26 \\
\hline Matched unrelated & 10 & 2 & 12 \\
\hline Mismatched unrelated & 6 & 1 & 3 \\
\hline \multicolumn{4}{|l|}{ Days to neutrophil $>0.5 \times$} \\
\hline $10^{9} / \mathrm{L}$ median (range) & $13(8-45)$ & $11(11-18)$ & $12(9-25)$ \\
\hline \multicolumn{4}{|l|}{ Acute GVHD } \\
\hline None & 38 & 6 & 24 \\
\hline Grades I-II de novo/after DLI & $4 / 2$ & $1 / 0$ & $9 / 3$ \\
\hline \multicolumn{4}{|l|}{ Grades III-IV de novo/after } \\
\hline DLI & $2 / 6$ & $0 / 1$ & $2 / 3$ \\
\hline
\end{tabular}

AML indicates acute myeloid leukemia; ALL, acute lymphocytic leukemia; MDS, myelodysplastic syndrome; CML, chronic myeloid leukemia; CLL, chronic lymphocytic leukaemia; HD, Hodgkin disease; NHL, non-Hodgkin disease.

${ }^{*}$ The differences between these subgroups were not significant $(P>.05)$.

marrow graft from a UD. Eighteen of these patients received a UD graft as a second transplant.

\section{CMV infection}

Overall, 51 (50\%) patients developed a CMV infection (Table 2). The median time to first CMV infection was 27 days (range, 7-240 days) after transplantation, with all but one initial episode occurring before 100 days. Forty-five $(90 \%)$ of these patients showed evidence of CMV infection before 35 days after transplantation. There was no impact of donor type or previous transplant on the time to CMV infection.

\section{Recipient-donor serostatus}

The probability of developing a CMV infection among CMV-HR and CMV-IR patients was $84.8 \%$ (95\% CI, 75.0\%-94.6\%; Figure 1). There was no difference in the demographic profiles of CMV risk groups. There was no effect of the donor status on the incidence of CMV reactivation in CMV-HR patients. The reactivation rate in patients with seropositive and seronegative donors was $92 \%$ and $78 \%$, respectively. Fifty percent of the CMV-IR patients had evidence of CMV infection. Of the 41 CMV-LR patients, 25 were monitored for CMV infection and only one patient was documented to have CMV infection.

\section{Type of transplant}

Eighty-four percent (16 of 19) of the CMV-R UD transplant recipients reactivated CMV compared to $85.3 \%$ (35 of 41) of

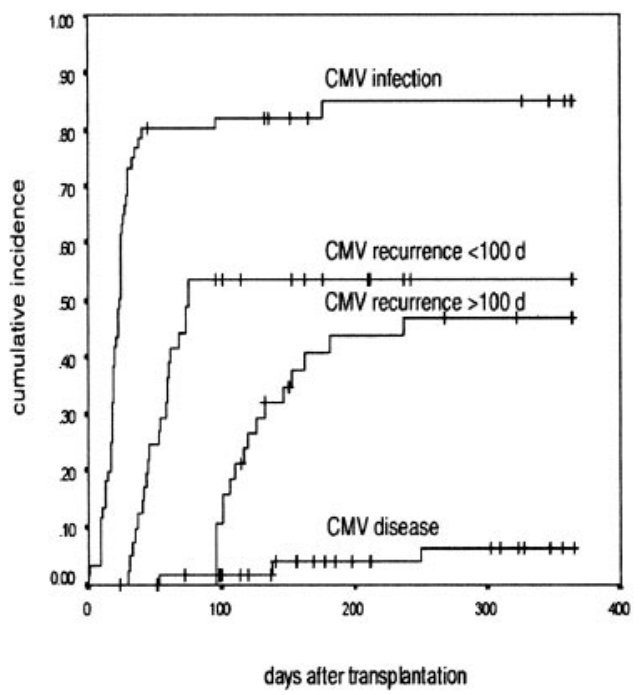

Figure 1. CMV infection after nonmyeloablative transplantation. A Kaplan-Meier graph shows the probability of developing CMV infection, CMV recurrence before and after 100 days, and CMV disease in the patients at risk of CMV disease after undergoing nonmyeloablative transplant. The cumulative incidence is in the $y$-axis and the time after transplantation is in the $\mathrm{x}$-axis.

sibling donor transplants among CMV-HR and CMV-IR patients. Reactivation was equally frequent among those patients receiving (94\%) or not receiving (85\%) a second transplant, irrespective of the source of the graft.

\section{Preemptive therapy}

GCV was used as first-line therapy in 44 episodes and foscarnet $(n=5)$ or cidofovir $(n=2)$ was used in others due to reactivation occurring in the pre-engraftment period. In 11 patients the treatment was changed, either due to nonresponse by 2 to 3 weeks or drug toxicity. The median duration of treatment for the first episode was 21 days (range, 10-57 days). There was a trend toward more prolonged therapy for UD transplants (median 27 days versus 21 days for matched related transplants, $P=.06$ ).

\section{Recurrence of CMV infection}

Forty-one of the 51 patients who had an initial episode of CMV infection were evaluable for recurrence because 10 patients were excluded from the analysis (death, 3; cytotoxic T-lymphocyte [CTL] therapy, 5; and inadequate follow-up, 2). The overall probability of recurrence was $71.3 \%$ (95\% CI, 56.7\%-85.8\%). Seventeen patients had 2 episodes of reactivation, with 11 patients having 3 or more episodes.

The probability of recurrence of CMV infection before 100 days after transplantation was $53.6 \%$ (95\% CI, 41.8\%-65.4\%; Figure 1). This developed at a median of 59 days (range, 35-80 days) after transplantation (Table 2). For recurrent infections before 100 days, the median overall duration of therapy was 15 days (range, 7-35 days).

Table 2. Recipient and donor CMV serostatus and outcome following nonmyeloablative transplantation among patients at risk of CMV disease*

\begin{tabular}{|c|c|c|c|c|c|c|}
\hline \multirow[b]{2}{*}{$\begin{array}{c}\text { CMV } \\
\text { serostatus }\end{array}$} & \multirow[b]{2}{*}{$\begin{array}{l}\text { CMV infection } \\
(\%)\end{array}$} & \multirow[b]{2}{*}{$\begin{array}{c}\text { Recurrence }<100 \mathrm{~d} \\
(\%)\end{array}$} & \multirow[b]{2}{*}{$\begin{array}{c}\text { Recurrence }>100 \mathrm{~d} \\
(\%)\end{array}$} & \multirow[b]{2}{*}{$\begin{array}{c}\text { CMV disease } \\
(\%)\end{array}$} & \multicolumn{2}{|c|}{ Nonrelapse mortality } \\
\hline & & & & & $\begin{array}{c}100 \mathrm{~d} \\
(\%)\end{array}$ & $\begin{array}{c}\text { Overall } \\
(\%)\end{array}$ \\
\hline $\mathrm{R}^{+} / \mathrm{D}^{+}$ & $35 / 38(92)$ & $16 / 27(59)$ & $9 / 24(41)$ & 2/38 (5.2) & $3(7.8)$ & $7(18.4)$ \\
\hline $\mathrm{R}^{+} / \mathrm{D}^{-}$ & $11 / 14(78)$ & $4 / 9(44)$ & $5 / 9(55)$ & $1 / 14(7.1)$ & $1(7.1)$ & $3(21.4)$ \\
\hline $\mathrm{R}^{-} / \mathrm{D}^{+}$ & $4 / 8(50)$ & $1 / 4(25)$ & $2 / 4(50)$ & $0 / 8(0)$ & $1(12.5)$ & $1(12.5)$ \\
\hline
\end{tabular}

${ }^{*}$ The differences between these subgroups were not statistically significant $(P>.05)$. 
From www.bloodjournal.org at UCL Library Services on August 20, 2008. For personal use only.

Table 3. Risk factors for recurrence of CMV infection following nonmyeloablative transplantation

\begin{tabular}{|c|c|c|c|c|c|c|}
\hline \multirow[b]{2}{*}{ Variable } & \multicolumn{2}{|c|}{ Reactivation $<100 \mathrm{~d}$} & \multicolumn{2}{|c|}{ Reactivation $>100 \mathrm{~d}$} & \multicolumn{2}{|c|}{ Overall reaction } \\
\hline & $\operatorname{OR}(P)$ & $95 \% \mathrm{Cl}$ & $\mathrm{OR}(P)$ & $95 \% \mathrm{Cl}$ & $\mathrm{OR}(P)$ & $95 \% \mathrm{Cl}$ \\
\hline \multicolumn{7}{|l|}{ Unrelated donor } \\
\hline Univariate & $3.3(0.005)^{*}$ & $1.4-7.8$ & $3.5(0.01)^{*}$ & $1.3-9.6$ & $3.5(0.002)^{*}$ & $1.5-7.9$ \\
\hline Multivariate & Not done & & $2.4(0.2)$ & $0.57-10.2$ & $7.5(0.001)^{*}$ & $2.1-26.9$ \\
\hline \multicolumn{7}{|c|}{$\mathrm{CD}{ }^{+} \mathrm{T}$-cell count at $3 \mathrm{mo}$} \\
\hline Univariate & $0.9(0.2)$ & $0.9-1.0$ & $0.98(0.04)^{*}$ & $0.96-0.99$ & $0.99(0.08) \dagger$ & $0.97-1.0$ \\
\hline Multivariate & Not done & & $0.98(0.08) \dagger$ & $0.96-1.0$ & $0.98(0.06) \dagger$ & $0.98-1.0$ \\
\hline \multicolumn{7}{|c|}{ Duration of previous episode(s) } \\
\hline Univariate & $0.9(0.1)$ & $0.9-1.0$ & $1.03(0.01)^{\star}$ & $1.0-1.06$ & $0.99(0.6)$ & $0.95-1.02$ \\
\hline Multivariate & Not done & & $1.03(0.2)$ & $0.97-1.09$ & Not entered & \\
\hline
\end{tabular}

*Significant value $(P<.05)$.

†Borderline significance $(P<.1)$.

Eleven of the 13 evaluable UD graft recipients had recurrence by 100 days compared to only 11 of 28 evaluable recipients of matched family donor grafts $(P=.01)$. There was no impact of patient age, donor-recipient serostatus (Table 2), previous transplant, or duration of the first episode on the recurrence of CMV infection before 100 days.

\section{Late CMV infection}

Sixty-one patients were potentially evaluable for late infection due to either their CMV risk status $(\mathrm{n}=60)$ or CMV infection in CMV-LR patients $(n=1)$, but 23 individuals were excluded from final analysis (death, 5; retreatment of relapse, 3; CTL therapy, 6; inadequate follow-up, 4; not monitored due to lack of reactivation before 100 days, 5). The probability of late reactivation was $46.6 \%$, (95\% CI, 30.3\%-62.9\%) in the 38 evaluable patients at a median of 122 days (range, 100-240 days; Figure 1). All except one individual had an initial episode of CMV infection before 100 days. The number of reactivation episodes ranged from 1 to 4 per patient (median, 1 episode). The total duration of antiviral therapy for all the episodes per patient varied between 10 and 121 days (median, 18 days).
Late CMV infection occurred in 7 of the 10 evaluable patients who had a UD transplant, compared to 10 of 28 evaluable patients following sibling transplants $(P=.01)$. Recurrence before 100 days was not a risk factor for late infection but the total duration of CMV-PCR positivity for all episodes before 100 days (median 46 versus 22.5 days in patients without late reactivation, $P=.01$ ) correlated significantly with late CMV infections (Table 3).

\section{Immune reconstitution}

The pattern of recovery of the T-cell subsets was serially monitored in 48 patients. CD4 recovery was slow across all the subgroups, with none achieving a normal $\mathrm{CD} 4^{+} \mathrm{T}$-cell count at 1 year after transplantation. Figure 2 depicts the recovery of $\mathrm{CD} 4^{+}$and $\mathrm{CD} 8^{+} \mathrm{T}$ cells in unrelated and matched family donors. This was similar in the 2 groups. All the symptomatic infections occurred when the $\mathrm{CD}^{+}{ }^{+}$count was below $200 \mathrm{CD}^{+}{ }^{+} \mathrm{T}$ cells $/ \mu \mathrm{L}$. The value of 200 $\mathrm{CD}^{+}{ }^{+} \mathrm{T}$ cells $/ \mu \mathrm{L}$ was achieved at a median of 9 months $(95 \% \mathrm{CI}$, 7.06-10.94). The CD4 ${ }^{+} \mathrm{T}$-cell count was significantly delayed in patients with late CMV reactivation (mean $\pm \mathrm{SD}=36.1 \pm 30.1$ / $\mu \mathrm{L})$, compared to patients not experiencing a late reactivation
A

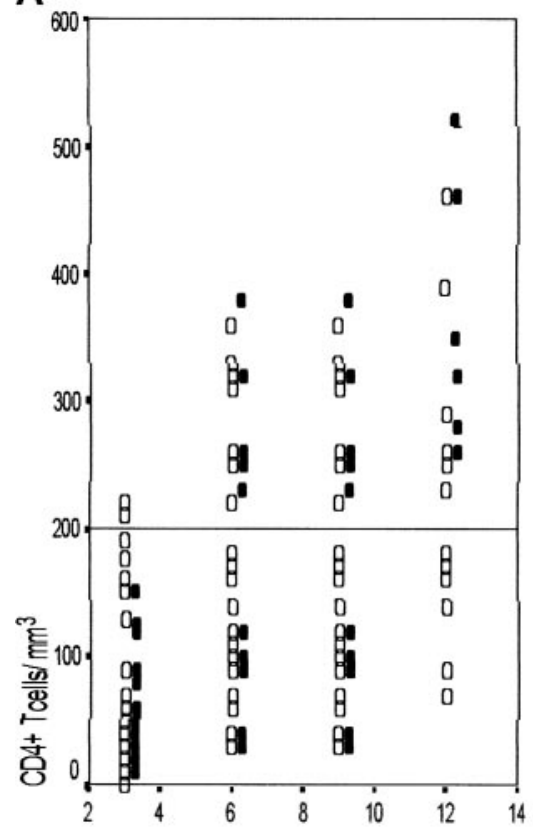

months after transplantation

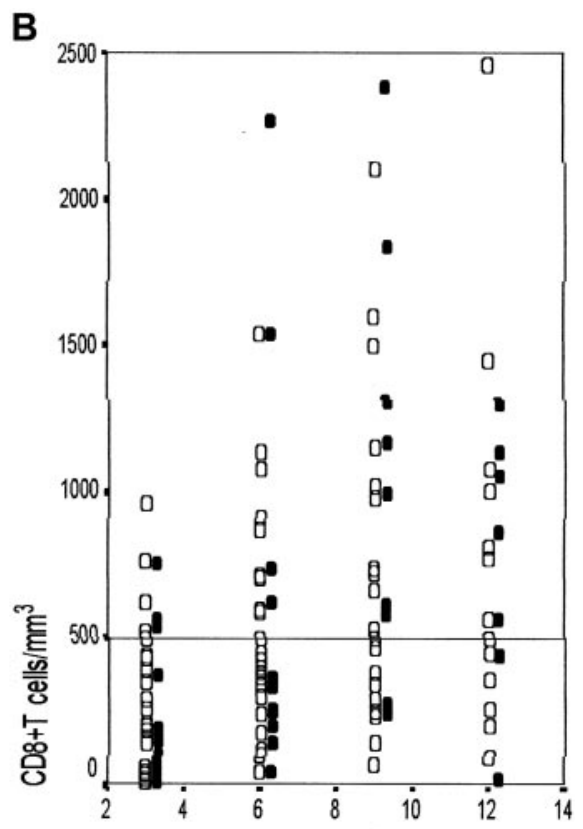

months after transplantation
Figure 2. Immune reconstitution following nonmyeloablative transplantation. Scatterplots showing the $\mathrm{CD}^{+} \mathrm{T}$-cell (A) and CD8 ${ }^{+} \mathrm{T}$-cell (B) counts at 3-month intervals after the procedure in patients undergoing nonmyeloablative transplantation. The $\mathrm{CD} 4^{+} / \mathrm{CD} 8^{+} \mathrm{T}-$ cell counts are shown in the $y$-axis and the time after transplantation is in the $\mathrm{x}$-axis. represent UD transplant recipients and $\square$ represent matched family donor transplants. In panels $A$ and $B$ the dotted lines represent the cut-off values for CD4 ${ }^{+} T$ cells at $200 / \mu \mathrm{L}$ and $\mathrm{CD} 8^{+} \mathrm{T}$ cells at $500 / \mu \mathrm{L}$. 
Table 4. Causes of mortality following nonmyeloablative transplantation*

\begin{tabular}{lccc}
\hline \multicolumn{1}{c}{ Cause of death } & CMV-HR & CMV-IR & CMV-LR \\
\hline Infections (non-CMV) & 2 & 0 & 5 \\
CMV-related & 5 & 0 & 0 \\
Definite/possible & $3 / 2$ & & \\
GVHD & 0 & 1 & 3 \\
Idiopathic pneumonia & 1 & 0 & 2 \\
Neurologic complications & 1 & 0 & 1 \\
Regimen-related toxicity & 1 & 0 & 1 \\
Relapse & 6 & 1 & 0 \\
\hline
\end{tabular}

*The differences between these subgroups were not statistically significant $(P>.05)$

(mean $\pm \mathrm{SD}=98.1 \pm 61.6 / \mu \mathrm{L} ; P=.04$ ). However, immune reconstitution data were available for only 48 patients and this analysis should be interpreted with caution because of the small numbers in each subgroup.

\section{Risk factors for CMV recurrence}

Recurrence of CMV infection was common. The dependent variables analyzed were recurrence before 100 days, recurrence after 100 days, and overall recurrence. UD transplantation was a significant risk factor for all 3 groups on univariate analysis, and this remained the single most important risk factor for overall reactivation and reactivation before 100 days on multivariate analysis. The other risk factors for late recurrence were low $\mathrm{CD}^{+}$ $\mathrm{T}$ cells at 3 months and the duration of previous CMV episodes before 100 days. However, on multivariate analysis, only the CD4 ${ }^{+}$ T-cell count at 3 months maintained borderline significance $(P=.08)$ in the subgroup of 23 patients on whom the data were available. This could be as a result of the smaller sample size, when CD4 ${ }^{+}$T-cell count was considered.

\section{CMV disease and death}

Three patients had definite CMV disease (actuarial probability $6.4 \%$ among CMV-HR and CMV-IR patients at 12 months after transplantation); 2 had pneumonia on days +40 and +100 and 1 had gastrointestinal involvement on day +230 after transplantation (Figure 1). In addition, there were 2 possible CMV deaths. One patient had progressive interstitial pneumonia on day +50 , with persistent CMV-PCR positivity with no other etiology. Another patient developed severe liver failure on day +35 following CMV reactivation. A tissue diagnosis was precluded in both these patients due to severe coagulopathy and permission was not obtained for necropsy.

All 5 patients died within 4 weeks of presentation. Two of these 5 deaths occurred after 100 days and both were associated with grade III to IV GVHD following infusion of donor lymphocytes. All 5 patients were CMV-HR and 3 of them received grafts from family donors.

\section{GVHD}

Eighteen patients (17.8\%) developed acute GVHD de novo, with grade III to IV GVHD in only 4 patients (3.9\%). Thirty patients received DLI and half of them developed GVHD. Grade III to IV GVHD developed in 10 patients after DLI (Table 1). Thus, overall, 33 patients developed GVHD and 14 had grade III to IV disease. Of the 4 deaths attributable to GVHD, only 2 occurred de novo. Chronic extensive GVHD developed in 4 patients, but in 3 of them it occurred after DLI. There was no influence of the donor type on the occurrence of GVHD either de novo or after DLI. No correlation was detected between CMV risk status or CMV infection and GVHD.

\section{Nonrelapse mortality and overall survival}

Twenty-three patients died of nonrelapse causes at a median follow-up of 15 months. Infections accounted most of the deaths with CMV being the single most important infectious cause for nonrelapse mortality (Table 4). Death within the first 100 days due to regimen-related causes was observed in 2 patients only. Two more patients died from neurologic complications and 2 CMV-LR patients died of idiopathic pneumonitis. No single variable was identified as a risk factor for nonrelapse mortality.

The probability of 100 -day mortality was $12.1 \%$ (95\% CI, $5.7 \%-18.5 \%$ ) and the actuarial probability of overall nonrelapse mortality at 12 and 18 months was $22.7 \%$ (95\% CI, $14.0 \%-31.4 \%$ ) and $27.8 \%$ (95\% CI, $17.2 \%-38.4 \%$ ), respectively. There was no difference in the nonrelapse mortality depending on CMV infection (Figure 3A) or serostatus (Table 2).

The actuarial probability of overall survival at 12 months and 18 months was $71.3 \%$ (95\% CI, 61.7\%-80.59\%) and 65\% (95\% CI,
Figure 3. Nonrelapse mortality and overall survival following nonmyeloablative transplantation. A KaplanMeier graph shows the cumulative probability of $(A)$ nonrelapse mortality in patients with $(\mathrm{n}=51$, events 10$)$ and without CMV infection ( $\mathrm{n}=50$, events $13 ; P=.44)$ and $(B)$ overall survival in patients with $(n=51$, events 13) and without CMV infection $(n=50$, events 17 $P=.37$ ). The patients with CMV infection are represented by the broken line and those without CMV infection are shown as a solid line.
A

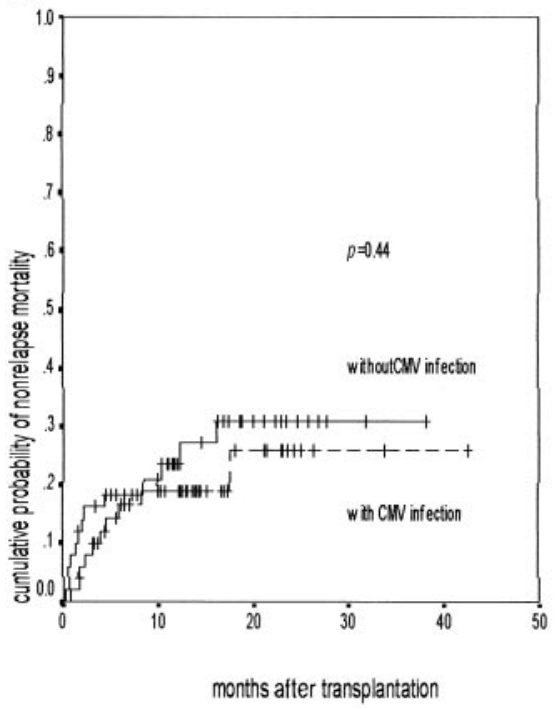

B

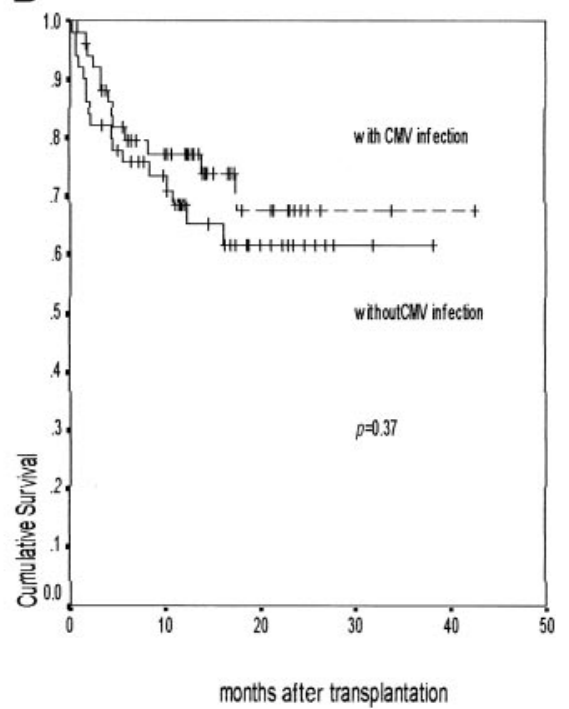


54.1\%-75.9\%), respectively. Survival was similar in patients infected with CMV or not infected (Figure 3B). The survival for UD transplant recipients was also similar to those receiving a matched family donor graft.

\section{Discussion}

The incidence and outcome of CMV infections after nonmyeloablative transplantations are unknown. Our study has shown a very high incidence of CMV infection with very early reactivation in this cohort of patients receiving Campath-1H in vivo. Such high rates of reactivation have not been reported following conventional transplants from matched family donors. ${ }^{21,22}$ In one other report on infections after nonmyeloablative transplants 65\% (13 of 20) of the patients developed CMV infections following antithymocyte globulin-based conditioning. ${ }^{23}$ Recent reports have suggested that the incidence of CMV reactivation after fludarabine and low-dose total body irradiation-based conditioning schedules seemed to be similar or slightly lower, compared to conventional transplants. ${ }^{24,25}$

Reactivation of CMV among the UD graft recipients, which comprised a third of the CMV at-risk group, was comparable to that following conventional T cell-depleted transplants. ${ }^{20}$ However, the UD transplant group was characterized by a high incidence of recurrent CMV infection. The kinetics of the T-cell subset recovery was not slower for UD transplant recipients, but it is possible that HLA class I/II microvariant disparities could have predisposed to more specific defects of virus-specific CTL recovery. However, although the comparisons between the donor types are relevant, the statistical power for these data are limited due to the small numbers involved in subgroup analysis.

Late reactivation occurred in just less than half of the patients at risk. Few studies ${ }^{18}$ have carried out extended CMV surveillance beyond 100 days and CMV infection beyond this period is usually identified only when symptomatic. A longer persistence of CMV infection or frequent reactivation before 100 days predisposed to late infections, particularly in UD transplant recipients. Previous studies in transplant recipients ${ }^{10,26}$ have demonstrated the pivotal role of $\mathrm{CD}^{+} \mathrm{T}$ cells in the development of CMV infection as well as disease. An analysis of this subgroup revealed a correlation between $\mathrm{CD} 4^{+}$T-cell counts at 3 months and late CMV infections. However, because of the small number of patients the data should be interpreted with caution.

Despite such a high incidence of both early and late CMV reactivation, the incidence of definite $\mathrm{CMV}$ disease was limited to $6 \%$ among the CMV at risk group. This could be due to several factors. First, the intensive and extended preemptive strategy was probably crucial in preventing CMV disease. With $45 \%$ of the patients experiencing late reactivation, it is not unreasonable to assume that the occurrence of late CMV disease was aborted to some extent by the use of extended preemptive therapy. However, intermittent preemptive therapy was not sufficient to prevent recurrent reactivation, particularly among UD transplants.

Second, severe GVHD and its treatment, which are the most important factors in the predisposition to CMV disease, ${ }^{4-6,8,9}$ were observed de novo in only $4 \%$ of the patients in this study. Both of the patients with late CMV disease in our cohort had severe GVHD and all the episodes of CMV disease were fatal. The low incidence of GVHD probably influenced the outcome of recurrent CMV infections occurring in this high-risk population.

On the other hand, the high rate of CMV and non-CMV infections in our study could be due to the use of Campath-1H in the conditioning regimen. Accurate assessment of the extent of T-cell depletion with Campath in vivo is not possible. Campath when used in vivo has been reported to be present in the circulation in sufficient amounts $(0.5-3.62 \mu \mathrm{g} / \mathrm{mL})$ for variable periods to cause antibody-dependent cell cytotoxicity of the graft depending on the dose and duration of treatment. ${ }^{27}$ The regimen was effective in reducing the incidence of GVHD, but Campath- $1 \mathrm{H}$ in vivo used in these patients may have had a more profound effect on immune reconstitution.

The kinetics of T-cell recovery in patients receiving related donor grafts was not different from that of the UD group. In addition, the majority of the CMV disease and death occurred in the related donor group. Moreover, CMV seropositivity of the donor did not reduce the incidence of CMV infection, recurrence, or disease in seropositive recipients. Thus, posttransplant immune reconstitution and its impact are unlikely to be different for related and unrelated donors if T-cell depletion is extensive. These findings have prompted us to initiate a study with de-escalation of the dose of Campath-1H in fully matched related and unrelated donors to determine the optimum balance between reduction of GVHD and immune reconstitution. Further detailed studies on immune reconstitution and infections are needed in these high-risk patients undergoing experimental conditioning strategies for allogeneic hematopoietic stem cell transplantation.

\section{Acknowledgments}

We thank the staff of the Therapeutic Antibody Centre, University of Oxford for their contributions to the production of Campath-1H antibody. Their work was supported by the United Kingdom Medical Research Council, Leukosite Inc, and the EP Abraham's Trust.

\section{References}

1. Ljungman $\mathrm{P}$, Engelhard D, Link $\mathrm{H}$, et al. Treatment of interstitial pneumonitis due to cytomegalovirus with ganciclovir and intravenous immune globulin: experience of European Bone Marrow Transplant Group. Clin Infect Dis. 1992;14:831835.

2. Avery RK, Adal KA, Longworth DL, Bolwell BJ. A survey of allogeneic bone marrow transplant programs in the United States regarding cytomegalovirus prophylaxis and pre-emptive therapy. Bone Marrow Transplant. 2000;26:763-767

3. Einsele $\mathrm{H}$, Ehninger $\mathrm{G}$, Hebart $\mathrm{H}$, et al. Polymerase chain reaction monitoring reduces the incidence of cytomegalovirus disease and the dura- tion and side effects of antiviral therapy after bone marrow transplantation. Blood. 1995;86: 2815-2820.

4. Boeckh M, Gooley TA, Myerson D, Cunningham T, Schoch G, Bowden RA. Cytomegalovirus pp65 antigenemia-guided early treatment with ganciclovir versus ganciclovir at engraftment after allogeneic marrow transplantation: a randomized double-blind study. Blood. 1996; 88:4063-4071.

5. Einsele $\mathrm{H}$, Hebart $\mathrm{H}$, Kauffmann-Schneider $\mathrm{C}$, et al. Risk factors for treatment failures in patients receiving PCR-based preemptive therapy for CMV infection. Bone Marrow Transplant 2000;25: 757-763.
6. Nguyen Q, Champlin R, Giralt S, et al. Late cytomegalovirus pneumonia in adult allogeneic blood and marrow recipients. Clin Infect Dis. 1999;28: 618-623.

7. Bacigalupo A, Mordini N, Pitto, A et al. Transplantation of HLA-mismatched CD34+ ${ }^{+}$selected cells in patients with advanced malignancies: severe immunodeficiency and related complications. $\mathrm{Br} \mathrm{J}$ Haematol. 1997;98:760-766.

8. Gor D, Sabin C, Prentice HG, et al. Longitudinal fluctuations in cytomegalovirus load in bone marrow transplant patients: relationship between peak virus load, donor/recipient serostatus, acute GVHD and CMV disease. Bone Marrow Transplant. 1998;21:597-605. 
From www.bloodjournal.org at UCL Library Services on August 20, 2008. For personal use only.

9. Nichols WG, Corey L, Gooley T, et al. Rising pp65 antigenemia during preemptive anticytomegalovirus therapy after allogeneic hematopoietic stem cell transplantation: risk factors, correlation with DNA load, and outcomes. Blood. 2001;97:867874.

10. Einsele H, Ehninger G, Steidle M, et al. Lymphocytopenia as an unfavorable prognostic factor in patients with cytomegalovirus infection after bone marrow transplantation. Blood. 1993;82:16721678.

11. Li CR, Greenberg PD, Gilbert MJ, Goodrich JM, Riddell SR. Recovery of HLA-restricted cytomegalovirus (CMV)-specific T-cell responses after allogeneic bone marrow transplant: correlation with CMV disease and effect of ganciclovir prophylaxis. Blood. 1994;83:1971-1979.

12. Giralt S, Estey E, Albitar M, et al. Engraftment of allogeneic hematopoietic progenitor cells with purine analog-containing chemotherapy: harnessing graft-versus-leukemia without myeloablative therapy. Blood. 1997;89:4531-4536.

13. Slavin S, Nagler A, Naparstek E, et al. Nonmyeloablative stem cell transplantation and cell therapy as an alternative to conventional bone marrow transplantation with lethal cytoreduction for the treatment of malignant and nonmalignant hematologic diseases. Blood 1998;91:756-763.

14. Kottaridis PD, Milligan DW, Chopra R, et al. In vivo Campath- $1 \mathrm{H}$ prevents graft-versus-host dis- ease following nonmyeloablative stem cell transplantation. Blood. 2000;96: 2419-2425.

15. Giralt S, Thall PF, Khouri I, et al. Melphalan and purine analog-containing preparative regimens: reduced-intensity conditioning for patients with hematologic malignancies undergoing allogeneic progenitor cell transplantation. Blood. 2001;97: 631-637.

16. McSweeney PA, Storb R. Mixed chimerism: preclinical studies and clinical applications. Bio Blood Marrow Transplant. 1999;5:192-203.

17. Barrett J, Childs R. Non-myeloablative stem cell transplants. Br J Haematol. 2000;11:6-17.

18. Peggs KS, Preiser W, Kottaridis PD, et al. Extended routine polymerase chain reaction surveillance and pre-emptive antiviral therapy for cytomegalovirus after allogeneic transplantation. $\mathrm{Br} \mathrm{J}$ Haematol. 2000;111:782-790.

19. Qamruddin AO, Oppenheim BA, Guiver M, Mutton KJ, Chopra R. Screening for cytomegalovirus $(\mathrm{CMV})$ infection in allogeneic bone marrow transplantation using a quantitative whole blood polymerase chain reaction (PCR) method: analysis of potential risk factors for CMV infection. Bone Marrow Transplant. 2001;27:301-306.

20. Small TN, Papadopoulos EB, Boulad F, et al. Comparison of immune reconstitution after unrelated and related T-cell-depleted bone marrow transplantation: effect of patient age and donor leukocyte infusions. Blood. 1999;93:467-480.

21. Manteiga R, Martino R, Sureda A. Cytomegalovi- rus pp65 antigenemia-guided pre-emptive treatment with ganciclovir after allogeneic stem transplantation: a single-center experience. Bone Marrow Transplant. 1998;22:899-904.

22. Meyers JD, Flournoy N, Thomas ED. Risk factors for cytomegalovirus infection after human marrow transplantation. J Infect Dis.1986;153:478-488.

23. Mohty M, Faucher $\mathrm{C}$, Vey $\mathrm{N}$, et al. High rate of secondary viral and bacterial infections in patients undergoing allogeneic bone marrow minitransplantation. Bone Marrow Transplant 2000; 26:251-255

24. Redy V, Pollock BH, Sharda S, et al. GVHD and $\mathrm{CMV}$ antigenemia after allogeneic peripheral blood stem cell transplantation: comparison between myeloablative and nonmyeloablative (mini) conditioning regimens. Blood. 2000;96 (suppl 1): 188a.

25. Junghanss $\mathrm{C}$, Boeckh $\mathrm{M}$, Carter R, et al. Incidence of herpesvirus infections following nonmyeloablative allogeneic stem cell transplantation. Blood 2000;96(suppl 1):188a.

26. Walter EA, Greenberg PD, Gilbert MJ, et al. Reconstitution of cellular immunity against cytomegalovirus in recipients of allogeneic bone marrow by transfer of T-cell clones from the donor. N Engl J Med. 1995;333:1038-1044.

27. Rebello P, Cwynarsky K, Varughese M. et al. Pharmacokinetics of Campath-1H in bone marrow transplant patients. Cytotherapy. 2001;3:261267. 